\title{
A Study of SARS-COV-2 Outbreaks in US Federal Prisons: the Linkage Between Staff, Inmate, and Community Transmission
}

Sherry Towers ( $\sim$ Sherry.Towers@iass-potsdam.de)

Institute for Advanced Sustainability Studies

Danielle Wallace

Arizona State University

Jason Walker

Arizona State University

John M. Eason

University of Wisconsin-Madison

Jake R. Nelson

The University of Texas at Austin

Tony H. Grubesic

The University of Texas at Austin

\section{Research Article}

Keywords: coronavirus, prisons, pandemic, SARS-COV-2, disease intervention strategies, decarceration, inmates, community

Posted Date: April 21st, 2021

DOl: https://doi.org/10.21203/rs.3.rs-384292/v1

License: (9) This work is licensed under a Creative Commons Attribution 4.0 International License. Read Full License 


\section{Abstract}

\section{Background}

Since the novel coronavirus SARS-COV-2 was first identified to be circulating in the US on January 20, 2020 , some of the worst outbreaks have occurred within state and federal prisons. The vulnerability of inmate populations, and the additional threats posed to the health of prison staff and the people they contact in surrounding communities underline the need to better understand the dynamics of transmission in the inter-linked inmate/staff/community sub-populations to better inform optimal control of SARS-COV-2.

\section{Methods}

We examined SARS-CoV-2 case data from 101 non-administrative federal prisons between 5/18/2020 to $01 / 31 / 2021$ and examined the per capita size of outbreaks in staff and inmates compared to outbreaks in the communities in the counties surrounding the prisons during the summer and winter waves of the SARS-COV-2 pandemic. We also examined the impact of decarceration, comparing inmate, staff and community SARS-COV-2 outbreak sizes during the winter wave to the summer wave.

\section{Results}

For both the summer and winter waves we found significant inter-correlations between per capita rates in the outbreaks among inmates, staff, and the community.

Over-all during the pandemic, per capita rates were significantly higher in inmates than in both the staff and community (paired Student's t-test $p=0.03$ and $p<0.001$, respectively). Per capita rates of inmate outbreaks depended significantly on security level, ranked from lowest to highest: High, Minimum, Medium, and Low security (population standardized Negative Binomial factor regression $p=0.02$ ).

Federal prisons decreased the number of inmates by a relative factor of $96 \%$ comparing the winter to summer wave (one SD range [90\%,102\%]). Prisons with relative decarceration below the mean had significantly lower ratio of winter to summer per capita rates in inmates, and also in the surrounding counties (two-sided Student's t-test $p=0.04$ and $p=0.02$, respectively). To our knowledge, this is the first analysis to examine the impact of decarceration on community rates of SARS-COV-2 transmission.

\section{Conclusions}

We found significant evidence of community/staff/inmate inter-linkage of SARS-COV-2 transmission. Decarceration ameliorates both prison and community SARS-COV-2 per capita rates. Further study is warranted to determine which control measures aimed at inmates and/or staff are most efficacious at preventing or controlling outbreaks.

\section{Background}


Since the novel coronavirus SARS-COV-2 was first identified to be circulating in the US on January 20, 2020 , some of the worst outbreaks have occurred within institutional settings such as homes for the elderly and in prisons and jails (1-5). For some time, prisons have been known facilitators of the spread of infectious diseases through multiple pathways $(6,7)$, including the architectural structure of shared space by those incarcerated (i.e., dining areas and living spaces), mass incarceration and subsequent over-crowding, and the social circumstances in the prison (i.e., unprotected sex and sexually transmitted diseases). For instance, tuberculous is rampant in US prisons, with approximately $25 \%$ of prisoners having a latent tuberculous infection (8-11). Moreover, between 29 to $43 \%$ of prisoners show evidence of previous Hepatis $\mathrm{C}$ infections (8-11)).

Similar to other infectious diseases, SARS-COV-2 infections among prisoners are common. To date, over 1 in 4 prisoners in the US have tested positive for SARS-COV-2 and over 2,400 have died (12). Controlling SARS-COV-2 infections in prisons is a critical part of "flattening the curve" (13), and outbreak and infection mitigation strategies used by prisons have largely been aimed at prisoners. However, a strict focus on inmates as carriers and transmitters of infectious diseases, including SARS-COV-2, means that containment efforts largely focused on that population ignore the potential that correctional staff have to influence infections both within and outside of prisons. Prisons are not closed systems, and while the prisoners have little contact with the outside world, correctional staff members move in and out of the prison to the community daily. As such, prison staff can both bring SARS-COV-2 into the prison, as well as take it into local communities (13).

In an attempt to control the spread of SARS-COV-2 in inmate populations, in May, 2020 the Federal Bureau of Prisons (BoP) instituted a halt on inmate visitation. In addition, many state and federal prisons decarcerated inmates at low-risk for re-offending in an effort to reduce crowding in the inmate population. Even despite these attempted control measures, the per capita rate of SARS-COV- 2 in incarcerated populations in the US was three to six times higher than the average rate in the main population $(1,3)$. However, to the authors' knowledge no study to date has compared rates of SARS-COV-2 in prisons to those in the communities that directly surround them, and the effects that control measures such as decarceration might have on community transmission of SARS-COV-2.

Here we examined the number of SARS-COV-2 cases in inmates and staff in 101 non-administrative federal prisons in the US, beginning May 18, 2020 (the date of the last BoP SARS-COV-2 directive in the spring) to Jan 31, 2021. We also examined the number of SARS-COV-2 cases in the counties surrounding the prisons, making this the first study of its kind to compare disease transmission in these linked subpopulations. We compared the per capita rates of the outbreaks in the three sub-populations, over-all during the pandemic and separately during the summer and winter waves. We examined how inmate population sizes and prison security level may have affected transmission rates, and also the effect of decarceration.

In the following sections we describe the sources of data and the statistical methods used, followed by a presentation of results and discussion. 


\section{Methods And Materials}

\section{Data}

Data on SARS-COV-2 incidence among inmates and staff in 121 federal prisons between 04/16/2020 (the date of the first recorded recovery in inmates or staff) and 1/31/2021 were obtained from the US Federal Bureau of Prisons website (14). We excluded 18 "administrative" facilities from consideration in the analysis, because several such facilities were medical centers (and thus specifically may have SARSCOV-2 positive inmates sent there, along with inmates with health conditions that might pre-dispose them to SARS-COV-2 infection) and others were transfer facilities. Privately run facilities in the federal system were also excluded because SARS-COV-2 incidence among staff in private facilities were not reported. Inmate to staff ratios and inmate population for the 103 remaining federal prisons were obtained from the Bureau of Prisons website (15). Inmate to staff ratios were not available for two prisons (FCI Marianna and USP Thompson) and thus they were excluded from further analysis, leaving 101 prisons. Unfortunately, for prisons that were part of larger multi-security-level complexes, the BoP only reported the inmate to staff ratios for the entire complex, which complicates calculation of per capita rates in staff. Thus, for portions of the analysis where the staff population needed to be known to calculate per capita rates we excluded prisons in complexes from our analysis, leaving a total of 65 prisons.

County level data on SARS-COV-2 incidence in the general population were obtained between 01/22/2020 and 1/31/2021 from the Johns Hopkins University Coronavirus Resource Center (16).

On May 8, 2020 the US Department of Justice released a memo to all federal Bureau of Prisons detention centers recommending the release to home confinement of at-risk prisoners who were at low risk of offending. A subsequent memo on May 14, 2020 describing testing protocols for new admissions, and another subsequent memo on May 18, 2020 recommended significant diminishment of movements between detention centers. To ensure uniformity of comparison of data from different prisons, we restricted the analysis to data collected on or after May 18, 2020.

Because this study used aggregated public data, it was determined not to constitute human subject research by the Arizona State University institutional review board.

It is important to note that the SARS-COV-2 data for inmates and staff in federal prisons qualitatively differ from the SARS-COV-2 data in the county main population in that the latter are the number of confirmed cases ("incidence") per day, whereas the former are the time series of the number of inmates and staff who have tested positive and are still positive ("prevalence"), along with the time series count of previously infected and subsequently recovered individuals and individuals who have died. Both of these time series were tallied by the BoP at various unevenly spaced time points, not necessarily daily. Unfortunately, data were not available on the total number of tests done per day in the BoP prison system. 
Over a long enough time period, the sum of the incidence is approximately equal to the total number of recovered individuals (for example, once an outbreak ends, the two numbers are equal). Thus, in portions of the analysis where we compared outbreak size in prisons to outbreak size in the county main population over a particular time period, we summed the incidence within the county, and compared to the number of recovered or dead inmates and staff over the same period. While not relevant to this analysis because we do not analyze temporal trends, it should be noted that the time series of the number of newly infected individuals lags the prevalence time series by the average infectious period divided by two (the infectious period for SARS-COV-2 is between 11 to 20 days (17)).

By May 18, 2020 all counties with federal prisons had recorded at least one SARS-COV-2 case, with the exception of Lassen County, CA where Herlong federal prison is located (the first case was recorded in that county on May 28,2020). Over half of federal prisons had no recorded cases in inmates or staff before that date. Between May 18, 2020 to Jan 31, 2021 just seven prisons accounted for over $50 \%$ of all cases in inmates and staff.

\section{Statistical methods}

Per capita rates

A commonly used statistic to assess relative outbreak size and temporal dynamics is the per capita number of cases (or deaths) in a population over some time period $(18,19)$. While often used, it must be cautioned that the detected number of cases in a population generally does not reflect the total number that actually occurred, particularly for diseases with a high asymptomatic rate and when testing availability is limited (18). In addition, comparing per capita rates across different locales is complicated by the fact that-testing availability and testing protocols may be quite different in the locales. "Different locales" can not only include different prisons, but also different states in which they (and their surrounding communities) reside.

In Fig. 1 we show the daily interpolated per capita prevalence rates of inmate, staff SARS-COV-2 infections summed over federal prisons, along with the per capita incidence rates in the counties surrounding the prisons. There is a distinct "two wave" pattern, particularly in the inmate and county populations, indicating that transmission rates changed in summer and winter, similar to the changes seen in the $2009 \mathrm{H} 1 \mathrm{~N} 1$ influenza pandemic (20). To ensure approximately constant transmission rates over the periods being considered, we thus divided the average peak time analysis into summer and winter waves, separated at the end of September, 2020.

Correlations and pair-wise comparisons

We examined the Spearman rho correlation between the per capita number of cases in federal nonadministrative prison inmate and staff populations, and to the per capita number of cases in the counties surrounding the prisons during the summer and winter waves. It should be noted here that any observed correlations between these quantities does not equate to one-way causation, and indeed the dynamics of 
disease transmission within the three inter-connected sub-populations are likely complex and not oneway.

We performed pairwise Student t-test comparisons of the three pairwise combinations of per capita rates in staff, inmates and the community, over-all, and during the summer and winter waves. While we examined per capita rates in this analysis in a manner similar to many other studies of SARS-COV-2 transmission in different settings, some caution in interpretation of results is warranted because of differing surveillance coverage in the different settings (21).

Dependence of per capita rates on prison population size and security level

We used population standardized Negative Binomial linear regression methods (see Appendix A) to assess the potential relationship of prison per capita rates of SARS-COV-2 infection in inmates and staff to prison population size and security level for both the summer and winter waves (and also over-all during the pandemic).

Assessing the effect of decarceration in reducing outbreak size

Comparing the average inmate populations during the winter wave compared to the summer wave, we found only modest levels of decarceration; on average inmate populations in non-administrative federal prisons during the winter wave were $96 \%$ of those during the summer wave (one SD range $[90 \%, 102 \%]$ ). Unfortunately, sparse SARS-COV-2 case numbers (particularly during the first wave) in the inmate and staff populations means that Least Squares linear regression methods (which assume the stochasticity in the data are Normal IID) are inappropriate for assessment of the effect of decarceration on the per capita number of cases during the second wave relative to the first. Thus, we used a two-sided Student's t-test to compare the per capita rates in winter minus the per capita rates summer,

$\Delta \rho=\rho_{\text {winter }}-\rho_{\text {summer }}$ for prisons with decarceration ratios below the mean to prisons with decarceration rates above the mean.

Over-all during the pandemic, we used population standardized Negative Binomial regression to examine the potential relationship of per capita rates to the ratio of the inmate populations on $01 / 31 / 2021$ to the population on $05 / 18 / 2020$, while taking into account prison security level and population size.

\section{Results}

\section{Correlations between per capita rates}

In Fig. 2 we show the correlogram showing the inter-correlation between the per capita rates in inmates and prison staff in the 101 non-administrative federal prisons, and in the population in the counties surrounding the prisons, during the summer and winter waves of the pandemic. During both waves significant positive inter-correlations are seen between the per capita rates in the sub-populations. 


\section{Pairwise comparison of per capita rates in outbreaks in staff, inmates, and the community}

All counties surrounding federal prisons had at least one SARS-COV-2 case during the first and second waves. In contrast, 8 (0) non-administrative federal prisons had zero cases in inmates and 16 (7) had zero cases in staff during the first (second) wave. During the first wave, 3 (9) prisons accounted for over $50 \%$ of the total number of cases in inmates (staff). During the second wave 13 (15) prisons accounted for over $50 \%$ of cases in inmates (staff).

We show the pairwise comparison of the per capita rates in the inmate, staff and county populations in Table 1. In general, per capita rates appeared to be highest in the inmate populations.

\section{Dependence of per capita rates on prison population size}

Using population standardized Negative binomial regression (see Appendix A), we linearly regressed the prisoner and staff SARS-COV-2 per capita rates on the logarithm of the prison population size for the 101 non-administrative federal prisons. Over-all during the pandemic and also during the winter wave, there was no significant dependence of the inmate per capita rates on the prison population size $(p=0.92$, and $p=0.33$, respectively). However, during the first wave there was significant positive dependence $(p=$ 0.002). There was no significant dependence of staff per capita rates on prison population size at any stage of the pandemic.

\section{Dependence of per capita rates on prison security level}

In Fig. 3 we show the per capita rates in staff and inmate populations, averaged within prison security level for the non-administrative federal prisons. Over-all during the pandemic, there was significant dependence of inmate per capita rates of SARS-COV-2 infection on prison security level (population standardized Negative Binomial factor regression $p=0.02$ ). The lowest per capita rates were in High and Minimum security prisons, and the highest were in Low security prisons.

During the summer wave (but not the winter wave), there was also significant dependence on security level for inmate per capita rates $(p<0.001)$. During the summer and winter wave and over-all during the pandemic, per capita rates in staff did not significantly depend on prison security level.

\section{Effect of decarceration on per capita rates in inmates, staff, and community}

When we compared the average inmate populations in non-administrative federal prisons during the winter wave compared to the summer wave, we found only modest levels of decarceration; on average inmate populations during the winter wave were $96 \%$ of those during the summer wave (one SD range $[90 \%, 102 \%])$. Using a two-sided Student's t-test, we compared the SARS-COV-2 per capita rates for

inmates, staff, and community in winter minus the per capita rates summer, $\Delta \rho=\rho_{\text {winter }}-\rho_{\text {summer }}$ for prisons with decarceration ratios below the mean to prisons with decarceration rates above the mean. For both inmates and the county population, $\Delta \rho$ was significantly lower for prisons with decarceration rates 
above the mean (two-sided t-test $\mathrm{p}=0.04$ and $\mathrm{p}=0.02$, respectively). For inmates, the average $\Delta \rho$ was $13 \%$ (26\%) for prisons with decarceration rates below (above) the mean. For the community, the average $\Delta \rho$ was $4 \%$ (5\%) for prisons with decarceration rates below (above) the mean. There was no significant difference for per capita rates in staff.

Using population standardized Negative Binomial regression we examined the potential relationship of per capita rates in inmates, staff and community to the change in inmate population from $05 / 18 / 2020$ to $01 / 31 / 2021$, while taking into account prison security level and average population size. We found no significant dependence of the per capita rates on the inmate population change for either inmates, staff, or the community.

\section{Discussion}

We examined the transmission of SARS-COV-2 in inmates and staff in non-administrative federal prisons from May 18, 2020 until January 31, 2021, and compared the patterns of transmission to those observed in the communities in the counties surrounding the prisons during the summer and winter waves of the pandemic. We found significant inter-correlations between per capita rates in outbreaks in inmates, staff and communities, and even inmate and surrounding community per capita rates were highly correlated, despite the fact that all inmate visitation had been halted over the time-frame of the data used in this study; transmission between those two sub-populations via the staff sub-population (which contacts both) is clearly a significant factor of SARS-COV-2 transmission in prisons, underlining the fact that these are inter-linked populations from the perspective of disease transmission, and a holistic approach needs

to be considered for infection control in prisons and the surrounding community that takes account of the complex dynamics between the three populations (7).

We examined the transmission of SARS-COV-2 in inmates and staff in non-administrative federal prisons from May 18, 2020 until January 31, 2021, and compared the patterns of transmission to those observed in the communities in the counties surrounding the prisons during the summer and winter waves of the pandemic. We found significant inter-correlations between per capita rates in outbreaks in inmates, staff and communities, and even inmate and surrounding community per capita rates were highly correlated, despite the fact that all inmate visitation had been halted over the time-frame of the data used in this study; transmission between those two sub-populations via the staff sub-population (which contacts both) is clearly a significant factor of SARS-COV-2 transmission in prisons, underlining the fact that these are inter-linked populations from the perspective of disease transmission, and a holistic approach needs to be considered for infection control in prisons and the surrounding community that takes account of the complex dynamics between the three populations (7).

During the second (but not first) wave of the pandemic we found that outbreaks in inmates had higher per capita rates than those in staff by a factor of 1.24 , suggesting greater disparity in the relative reproduction numbers in the two populations as the pandemic continued. It is unclear why this might be the case, although increased control measures among the community population (like mask wearing, 
school closures, limitations on restaurants and gyms, etc) may have decreased the per capita rates in the staff population to a level lower than they might have been otherwise, but meanwhile the prisoners were living in much the same environment as they had been during the summer wave (although it should be noted that most prisons reduced populations somewhat as the pandemic progressed through decarceration of low-risk inmates). These results are contrary to those of Puglisi et al (2020), who found that per capita rates of symptomatic infection were $40 \%$ higher in staff than inmates in an urban jail (22). Further study is warranted to determine the potential reasons for the disparity in results.

During the summer and winter waves of the pandemic both inmates and staff had significantly higher per capita rates of infection than outbreaks in the surrounding counties by over a factor of three. Other studies (eg; $(1,3)$ ) have examined SARS-COV-2 per capita rates in prisons compared to the main US population, but to the authors' knowledge, this is the first study to specifically examine the populations in the direct vicinity of prisons. In any study comparing prison and community per capita cases, it must be cautioned that differing SARS-COV-2 testing surveillance in prisons compared to the community might skew results $(1,23)$. Unfortunately, the BoP data do not include the number of SARS-COV-2 tests done per capita in staff and inmates, which could help to better understand the magnitude of the disparities in transmission compared to the community population.

We found significant dependence of inmate per capita rates on security level over-all, and during the summer wave of the pandemic. In general, as security levels rise inmates have less contact with other inmates, and inmate to staff ratios go down. We found that among Low, Medium, and High security prisons, per capita rates in inmates went down by security level, in broad agreement with the findings of (24). However, low per capita rates were also seen in Minimum security prisons, despite the high inmate to staff ratios. It is unclear why this is the case, but it may be because all inmates in federal Minimum security prisons work, whereas this is not true of the higher security prisons. Greater opportunity for inmate social activities in higher security prisons (particularly Low security prisons) perhaps may account for the disparities in transmission, but more study is needed of the daily activities of inmates in the prisons to determine where key disparities in transmission may be occurring, and how this might inform SARS-COV-2 control strategies.

We found that decarceration from the summer to winter wave of the pandemic had a significant impact on lowering rates of SARS-COV-2 infection in inmates, in concordance with the results of Vest et al (2020) who found that $85 \%$ decarceration in Texas state prisons was associated with significantly lower rates of SARS-COV-2 infection and death (24). Interestingly, we found that decarceration was also associated with lower SARS-COV-2 rates in the community. To the authors' knowledge, ours is the first study to examine this. The fact that decarceration actually decreased community risk of heightened SARS-COV-2 outbreaks is extremely interesting and again underlines the inter-connected nature of the inmate/staff/community populations(22), and how control measures aimed at one of those sub-populations can benefit the others. Further study is warranted to determine if this observation also holds for communities in the vicinity of state prisons. 
There were many issues affecting data quality in this analysis:

- Rather than publishing total number of newly identified cases per some time period (like day, week or month), the BoP instead publishes the number of people who have tested positive (and are still positive), along with the number recovered and number dead, and only does so sporadically and not at evenly spaced time points. The mismatch between having to compare prevalence in the prison populations to incidence in the community populations was a relatively minor complication to this analysis because we examine relatively long time periods. But the incidence vs prevalence reporting differences seriously complicate any potential future analysis of short term temporal trends in the data because incidence data lag that of prevalence by half the average infectious period. In addition, the daily reporting of community data, and irregular reporting of BoP data would also seriously complicate such an analysis. In general, the BoP surveillance data do not exhibit good epidemiological and public health practices in infectious disease reporting standards $(1,23)$. Greater governmental oversight and guidance on infectious disease surveillance and reporting for such institutions is warranted to make the data more useful towards informing outbreak control.

- While detailed temporal information is available on number of inmates per prison, estimates of staff come from one time point in mid 2020. This potentially creates some unreliability of our staff per capita rate assessments, if in fact the staff population sizes significantly varied over the time frame under consideration.

- Information of race and ethnicity of inmates in the federal prisons, and that of inmates testing positive for SARS-COV-2 are not available, but are potentially an important factor to take into account in any analysis of transmission disparities.

- The number of SARS-COV-2 cases in inmates and staff is published at the prison level, but for prisons that are part of larger multiple-security-level complexes, the BoP only publishes staffing information for the entire complex. Thus, per capita rates for staff could not be computed for those prisons, and they had to be excluded for parts of the analysis examining staff per capita rates. Should that data be made available, it would increase the sensitivity of the analysis to detecting potential dependence of staff per capita rates of infection on factors like security level.

- The BoP does not publish the number of tests performed, only the total number of positive tests (and deaths), and it is unclear what surveillance protocols were in place, and whether these differed by prison. For example, whether regular random testing of inmates or staff was performed to identify asymptomatic cases, or whether testing was solely performed on symptomatic individuals, or even just upon request.

Further study is needed to determine why just seven out of 101 non-administrative federal prisons accounted for the majority of SARS-COV-2 cases in inmates and staff, similar to the findings of a previous study of Texas prisons (24). While our studies shed some light on the factors that might be at play, an additional analysis of testing protocols should that data be made available would be useful. 
Further study is also needed to examine the primary directionality of transmission and determine whether or not peak times of outbreaks in communities tend to precede or lag peak times in inmate populations. High-risk sub-populations can form a "reservoir" of infection that can continue to re-infect the broader population even when strict control measures are in place (25). The highly complex dynamics of disease spread among inter-connected populations require mathematical models to assess the relative impacts of various proposed control measures, because some control measures aimed at one sub-population can lead to unexpectedly adverse outcomes for other populations. In future work, the authors will develop a model for inmate/staff/community SARS-COV-2 transmission and use it to perform in-vitro assessment of various potential control measures, including reducing crowding through decarceration, cohorting of inmates, quarantine, isolation, and decreased transmission through social distancing and use of PPE.

\section{Conclusions}

In the first study of its kind, we examined the transmission of SARS-COV-2 in inmates and staff in nonadministrative federal prisons and compared the patterns of transmission to those observed in the communities in the counties surrounding the prisons. We found significant inter-correlations between per capita rates in outbreaks in inmates, staff and communities. We also found that decarceration of inmates during the pandemic was associated with significantly lower rates of SARS-COV-2 infection in both inmates and members of the surrounding communities. These studies can help inform infectious disease response policies in prisons that help to mitigate rates of infection in inmates, staff, and the community at large.

\section{Declarations}

\section{Ethics approval and consent to participate:}

This study used aggregated publicly available data. The need for ethical approval and informed consent was waived by the institutional review board of Arizona State University.

\section{Consent for publication:}

Not applicable.

\section{Availability of data and materials:}

The SARS-COV-2 data sets analyzed during this study for prisons and community are publicly available at https://www.bop.gov/coronavirus and https://github.com/CSSEGISandData/SARS-COV-

2/tree/master/csse_SARS-COV-2_19_data/csse_SARS-COV-2_19_time_series (accessed March, 2021).

\section{Competing interests:}

The authors declare no competing interests. 


\section{Funding:}

Funding for this project comes from the National Science Foundation Sociology Program, Grant \#2032747, "Estimating the Reciprocal Relationship between COVID-19 Infections of Prisoners and Staff and Infections in the Surrounding Communities"

\section{Authors' contributions:}

All authors participated in the design of the study. ST, DW, and JW performed data collection. ST performed the statistical analyses, and ST and DW wrote the manuscript. All authors participated in reviewing and proofreading the manuscript.

\section{References}

1. Saloner B, Parish K, Ward JA, DiLaura G, Dolovich S. COVID-19 cases and deaths in federal and state prisons. Jama. 2020;324(6):602-3.

2. Franco-Paredes $\mathrm{C}$, Ghandnoosh N, Latif H, Krsak M, Henao-Martinez AF, Robins $M$, et al. Decarceration and community re-entry in the COVID-19 era. Lancet Infect Dis. 2020;

3. Jiménez MC, Cowger TL, Simon LE, Behn M, Cassarino N, Bassett MT. Epidemiology of COVID-19 Among Incarcerated Individuals and Staff in Massachusetts Jails and Prisons. JAMA Netw open. 2020;3(8):e2018851-e2018851.

4. Lemasters K, McCauley E, Nowotny K, Brinkley-Rubinstein L. COVID-19 cases and testing in 53 prison systems. Heal justice. 2020;8(1):1-6.

5. Oladeru OT, Tran N-T, Al-Rousan T, Williams B, Zaller N. A call to protect patients, correctional staff and healthcare professionals in jails and prisons during the COVID-19 pandemic. Heal justice. 2020;8(1):1-3.

6. Rubin AT. The prehistory of innovation: A longer view of penal change. Punishm Soc. 2018 Apr;20(2):192-216.

7. Franco-Paredes C, Jankousky K, Schultz J, Bernfeld J, Cullen K, Quan NG, et al. COVID-19 in jails and prisons: A neglected infection in a marginalized population. PLoS Negl Trop Dis. 2020;14(6):e0008409.

8. Bick JA. Infection control in jails and prisons. Clin Infect Dis. 2007;45(8):1047-55.

9. Dumont DM, Brockmann B, Dickman S, Alexander N, Rich JD. Public health and the epidemic of incarceration. Annu Rev Public Health. 2012;33:325-39.

10. Hammett TM, Roberts $C$, Kennedy S. Health-related issues in prisoner reentry. Crime Delinq. 2001;47(3):390-409.

11. Weinbaum CM, Sabin KM, Santibanez SS. Hepatitis B, hepatitis C, and HIV in correctional populations: a review of epidemiology and prevention. Aids. 2005;19:S41--S46.

12. The Marshall Project, https://www.themarshallproject.org, accessed March 1, 2021. 
13. Kinner SA, Young JT, Snow K, Southalan L, Lopez-Acuña D, Ferreira-Borges C, et al. Prisons and custodial settings are part of a comprehensive response to COVID-19. Lancet Public Heal. 2020;5(4):e188-e189.

14. US Federal Bureau of Prisons, https://www.bop.gov/coronavirus, accessed March 1, 2021.

15. US Federal Bureau of Prisons staffing, https://www.bop.gov/about/statistics/statistics_staff_staffing_ratios.jsp, accessed March 1, 2021.

16. Johns Hopkins Coronavirus Resource Center https://coronavirus.jhu.edu, accessed March 1, 2021.

17. Byrne AW, McEvoy D, Collins AB, Hunt K, Casey M, Barber A, et al. Inferred duration of infectious period of SARS-CoV-2: rapid scoping review and analysis of available evidence for asymptomatic and symptomatic COVID-19 cases. BMJ Open. 2020;10(8):e039856.

18. Larsen D, Dinero R, Asiago-Reddy E, Green H, Lane S, Shaw A, et al. A review of infectious disease surveillance to inform public health action against the novel coronavirus SARS-CoV-2. 2020;

19. Kremer H-J. International comparisons of coronavirus deaths and infections should be avoided if not adjusted per capita. Public Health. 2020;

20. Towers S, Feng Z. Pandemic H1N1 influenza: predicting the course of a pandemic and assessing the efficacy of the planned vaccination programme in the United States. Eurosurveillance. 2009;14(41):19358.

21. Team CDCC-19 R, Team CDCC-19 R, Team CDCC-19 R, Bialek S, Bowen V, Chow N, et al. Geographic differences in COVID-19 cases, deaths, and incidence-United States, February 12-April 7, 2020. Morb Mortal Wkly Rep. 2020;69(15):465-71.

22. Puglisi LB, Malloy GSP, Harvey TD, Brandeau ML, Wang EA. Estimation of COVID-19 basic reproduction ratio in a large urban jail in the United States. Ann Epidemiol. 2020;53:103-5.

23. Novisky MA, Narvey CS, Semenza DC. Institutional Responses to the COVID-19 Pandemic in American Prisons. Vict Offender. 2020;1-18.

24. Vest N, Johnson O, Nowotny K, Brinkley-Rubinstein L. Prison population reductions and COVID-19: a latent profile analysis synthesizing recent evidence from the Texas state prison system. J Urban Heal. 2020;1-6.

25. Avery C, Bossert W, Clark A, Ellison G, Ellison SF. An Economist's Guide to Epidemiology Models of Infectious Disease. J Econ Perspect. 2020;34(4):79-104.

26. Casella G, Berger RL. Statistical inference. Vol. 2. Duxbury Pacific Grove, CA; 2002.

27. Lloyd-Smith JO. Maximum likelihood estimation of the negative binomial dispersion parameter for highly overdispersed data, with applications to infectious diseases. PLoS One. 2007;2(2):e180.

28. Osgood DW. Poisson-based regression analysis of aggregate crime rates. J Quant Criminol. 2000;16(1):21-43.

\section{Table}


Due to technical limitations, table 1 docx is only available as a download in the Supplemental Files section.

\section{Figures}

\section{Non-administrative federal prisons $(\mathrm{N}=65)$}

\section{Daily prevalence (and county incidence):} May 18 to Jan 31

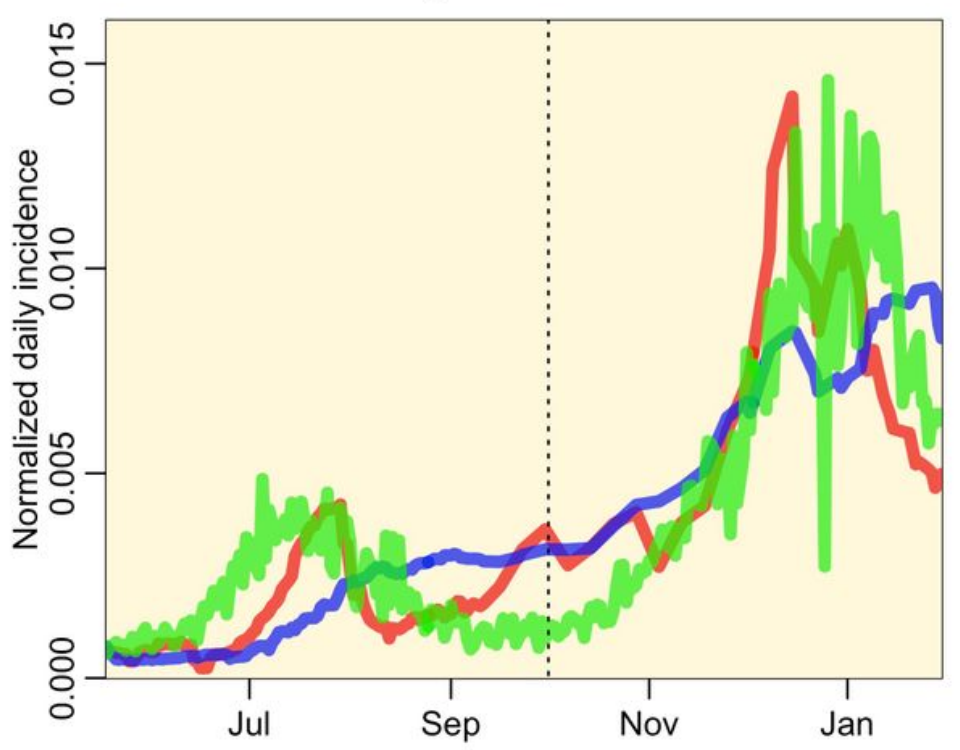

\section{Cumulative: May 18 to Jan 31}

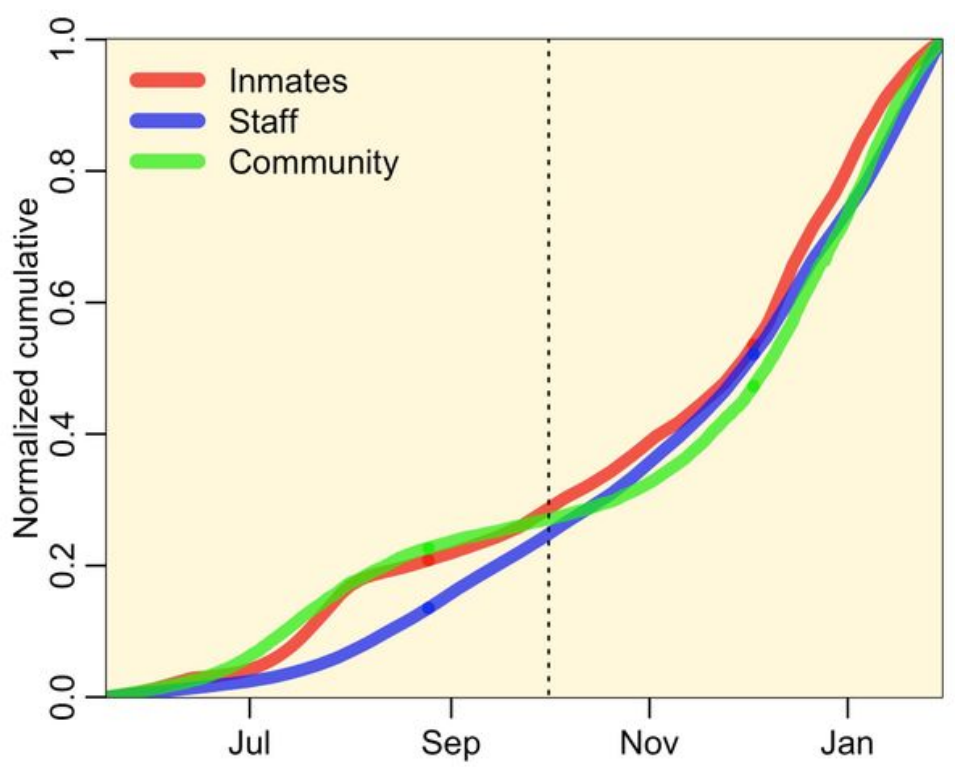

Figure 1

Per capita prevalence of SARS-COV-2 infections in inmate prison and staff summed over nonadministrative federal prisons for which the staff population is known, along with the per capita SARSCOV-2 incidence summed over the surrounding counties. For ease of comparison of the temporal patterns, the area under the curves are normalized to sum to one. The vertical black dotted line represents the nominal cut-off between the two waves at the end of September, 2020. Note that temporal patterns in inmates and staff per capita rates are dominated by just a few prisons; seven prisons account for over $50 \%$ of cases in both staff and inmates. 


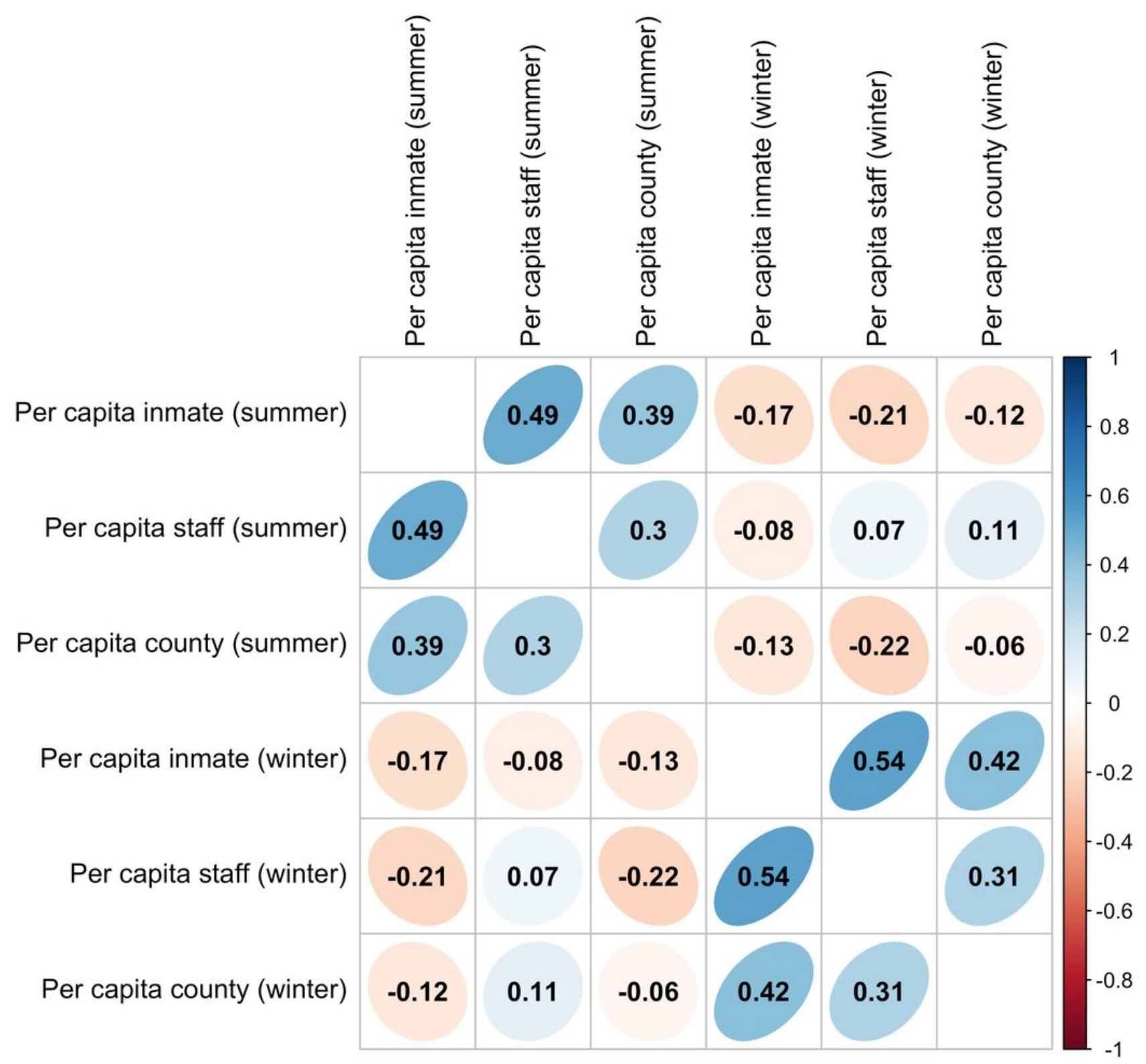

Figure 2

Correlations between per capita rates of SARS-COV-2 cases in inmates, staff in federal non-administrative prisons, and in the surrounding counties during the summer and winter waves of the pandemic. Absolute correlations larger than 0.20 are significant to $p<0.05$ (two-sided t-test). 


\section{Per capita rates}

Per capita cases staff: May 18, 2020 to Jan 31, 2021

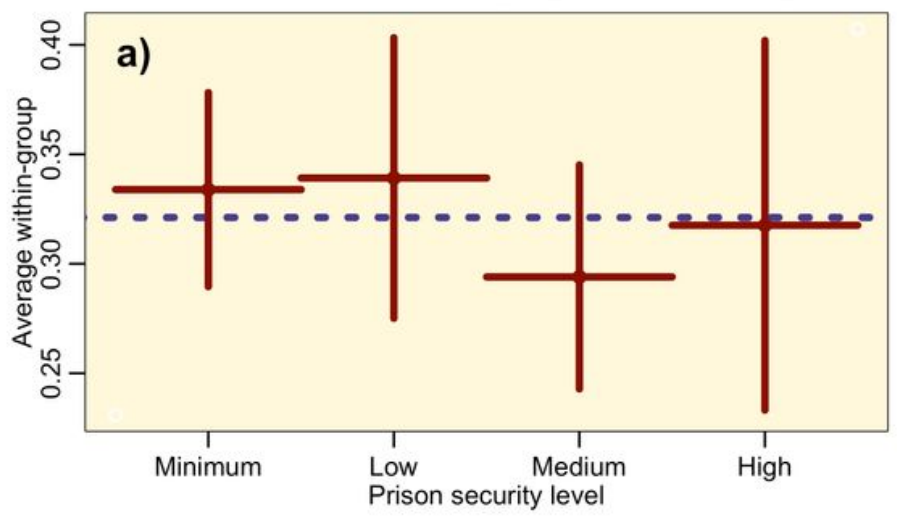

Per capita cases staff: Summer Wave

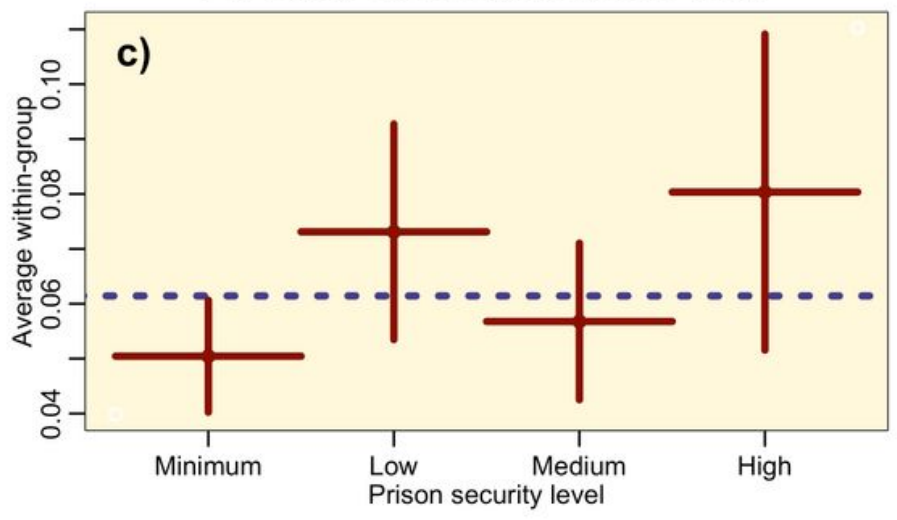

Per capita cases staff: Winter Wave

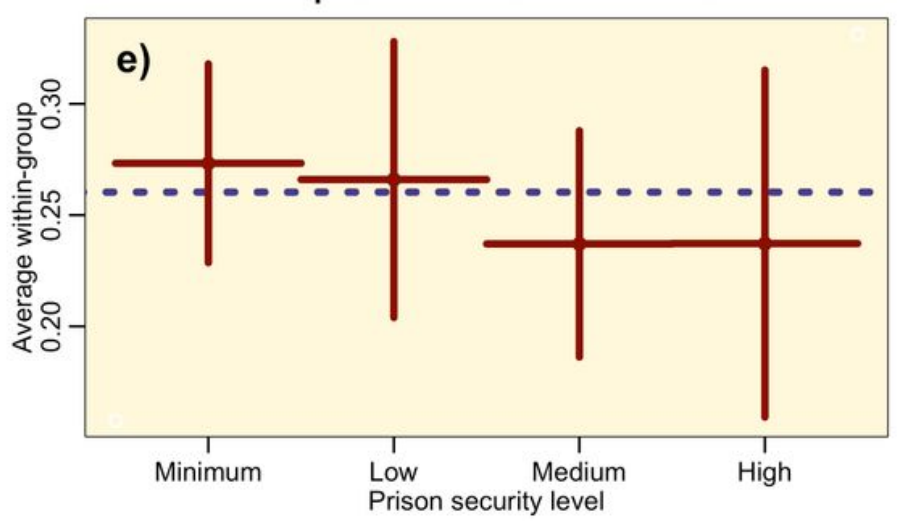

Per capita cases inmates: May 18, 2020 to Jan 31, 2021

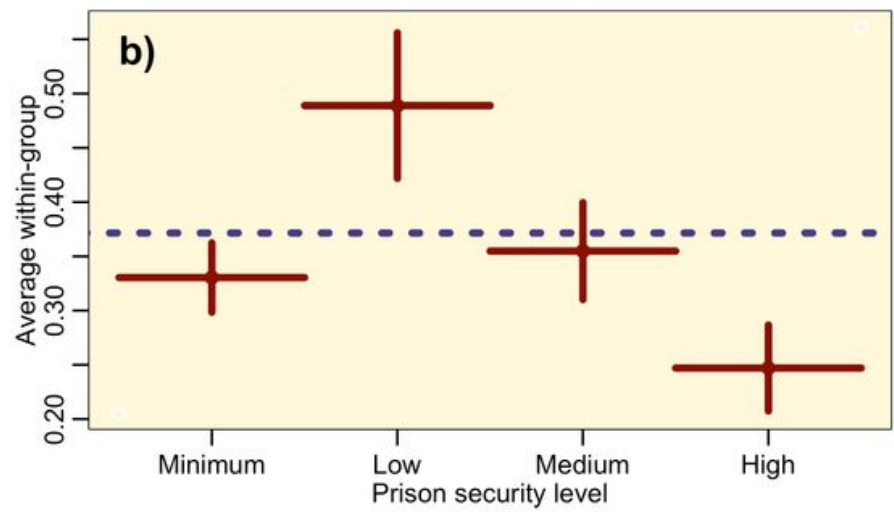

Per capita cases inmates: Summer Wave
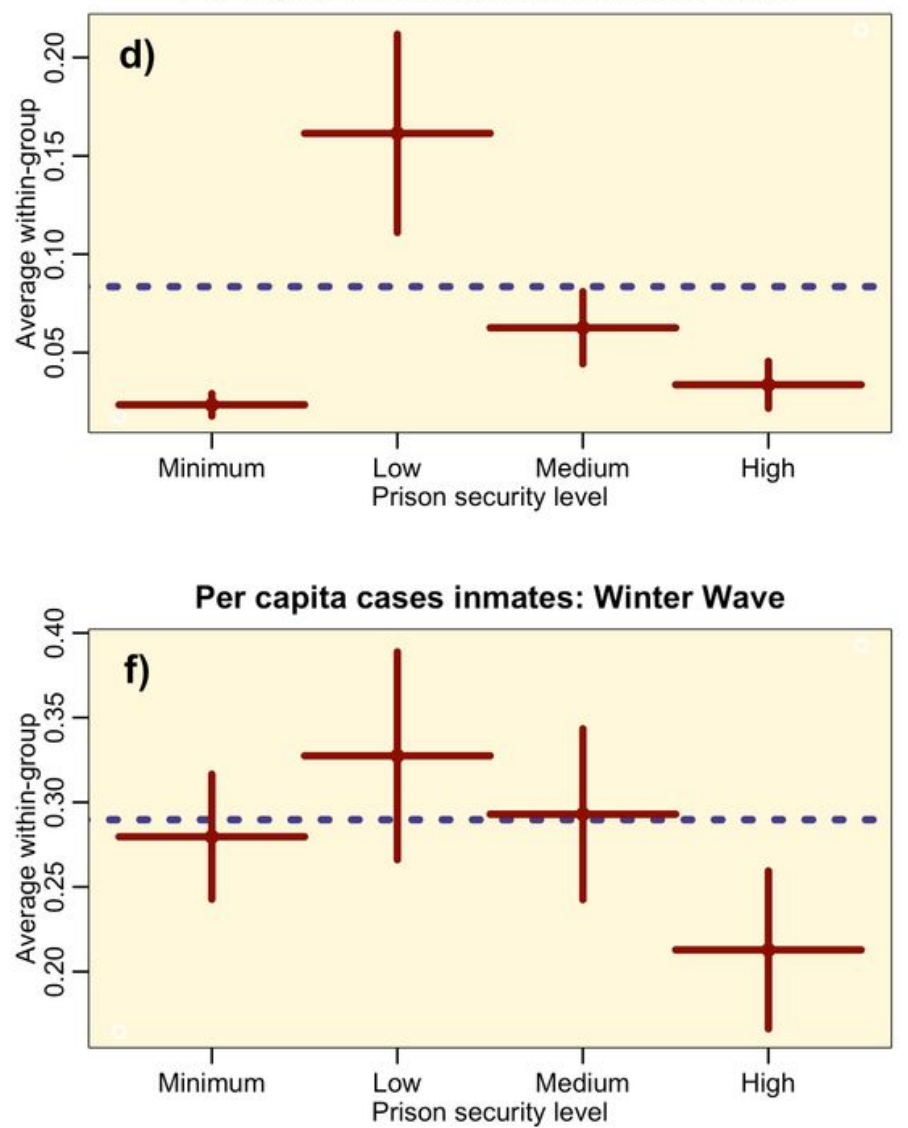

\section{Figure 3}

Distribution of mean SARS-COV-2 per capita rates in inmate and staff populations by security level for non-administrative federal prisons, over-all between May 18, 2020 to Jan 31, 2021 (top row) and for the summer and winter waves (second and third row). The vertical bars represent the standard error on the means. The dotted horizontal line represents the average over all security levels. Significant dependencies on security level are seen in b) and d) (population standardized Negative Binomial factor regression $\mathrm{p}<0.05$ in both cases). 


\section{Supplementary Files}

This is a list of supplementary files associated with this preprint. Click to download.

- Table1.docx

- statisticalanalysisreciprocalprisoncommunityappendix.docx 